\title{
Towards a Physics Based Model of Magnetic Barkhausen Noise in Steel
}

\author{
Thomas W. Krause*; Aroba Saleem, P. Ross Underhill \\ Department of Physics and Space Science, Royal Military College of Canada, Kingston, ON, Canada, K7K7B4
}

*Corresponding author: E-mail: thomas.krause@rmc.ca; Tel.: (001) 6135416000 Ext. 6415

DOI: 10.5185/amlett.2020.061526

Ferromagnetic iron based alloys are used in many important steel products including electrical steels, oil and gas pipelines, naval structures, aircraft landing gear and automotive components. Magnetic properties of these materials are vital for such applications as electrical motors and transformers, where they have direct implications for energy efficiency. Many inspection methods rely on measurement of magnetic response including magnetic particle inspection, magnetic flux leakage, metal magnetic memory, magnetic adaptive testing and magnetic Barkhausen noise (MBN). Understanding the dependence of magnetic properties on material condition is particularly important for detection of residual stress using MBN. This paper examines MBN response in a high strength naval steel, Q1N, undergoing magnetization, including domain structure, which is modified by application of tensile stress, and its interaction with pinning sites within grains, as provided by impurity elements and carbides, whose density is varied by isothermal tempering treatment. A physical basis for modifying domain structure and experimentally examining its interaction with changing pinning site density via temper embrittlement is presented to investigate stress dependent behaviour of MBN in such steel materials. The introduction of this experimental process will facilitate the botential of MBN sensing technologv for advanced monitoring of steel structure condition.

\section{Introduction}

Ferromagnetic iron based alloys, used to form steel products, are ubiquitous in our society, with critical applications arising in electrical steels, oil and gas pipelines, naval structures, aircraft landing gear and automotive components [1]. The magnetic properties of these materials can be critical for such applications as electrical motors and transformers, where magnetic properties have direct implications for energy efficiency. Inspection of ferromagnetic components using such tools as magnetic flux leakage (MFL) [2], and pulsed eddy current (PEC) [3], also require characterization of materials' magnetic properties such as permeability, to compensate for variations of this parameter on sizing of flaws or wall thickness, respectively. The presence of applied stress due to loading or the presence of residual stress due to manufacturing can also lead to changes in magnetic properties, which directly affect accuracy of inspection results [2].

Interplay between microstructure and material stress state, and formation of domains in ferromagnetic materials is important for application of magnetic materials such as used for electrical motors [4] and transformers [5], inspection of oil and gas pipelines [2], naval structures [6] and aircraft landing gear [7]. This interplay depends on length scales over which ferromagnetic domains form, and are defined by minimization of local magnetic energy elements, of which formation of demagnetizing fields plays the largest role [1]. Domain structure in the demagnetized state is itself the result of the underlying microstructure, crystallographic texture, inclusions, dislocations and residual stress. Under the action of an applied magnetic field or even applied physical stress, domain configurations are modified, as a result of overall energy minimization of the domain configuration [1] Insights into the formation and evolution of domain structures can be obtained using micromagnetic measurements that sense Barkhausen events [1].

Magnetic Barkhausen noise (MBN) is the result of abrupt changes in magnetic domain structure that arise when a time-varying magnetic field is applied to the sample. These abrupt changes include local changes in magnetization that occur when domain walls within domain structures overcome pinning barriers [8]. The pinning barriers may be the result of impurity elements, such as carbides, inclusions, and precipitates [1,8-10] and plastic deformation, which carry dislocations [11]. The domain structure is also affected by the presence of stress within the material [12,13], and effects of stress are modified by various material parameters such as grain size, crystallographic texture and microstructure [1]. This makes MBN sensitive to material conditions such as the presence of residual stress and plastic deformation, and properties of the material, such as hardness, strength and toughness [1]. The importance of a physical understanding of multiple parameters that affect the generation of Barkhausen noise has been emphasized for the characterization of material properties, such as the state of temper embrittlement, which refers to reduction of 


\section{Advanced Materials Letters www. vbripress.com/aml}

fracture toughness of alloy steels when heated or slowly cooled through the embrittling temperature range [14].

Magnetic Barkhausen noise (MBN) demonstrates sensitivity to material condition [1] and therefore, has been considered for non-destructive monitoring of ferromagnetic structures. However, MBN is sensitive to presence of impurity elements, carbides and inclusions, which pin domain walls [8], plastic deformation, which carry dislocations $[\mathbf{1 1}]$, the presence of residual $[\mathbf{1 5}, \mathbf{1 6}]$ and applied stress $[\mathbf{1 2 , 1 3}]$, and a large number of material parameters including steel surface condition [17], grain size [18], carbon content [19], crystallographic texture [20], and microstructure [21]. Form of the domain structure is determined by previous and current magnetization states. For example due to presence of remnant flux [22], magnetization rate [23], and peak magnetization level [24]. In particular, MBN is primarily a function of the ferromagnetic domain structure and its interaction with the energy landscape over which the domain walls move, and which produce the abrupt changes in magnetization associated with Barkhausen noise [1]. Energy landscape is determined by microstructure, texture, domain wall pinning sites and existing magnetization state within the material. Therefore, successful application of MBN technique as a non-destructive evaluation tool requires development of a fundamental understanding of the physical parameters that result in Barkhausen noise generation.

Studies of MBN emissions from carbon steels show an initially increasing amount of Barkhausen noise, associated with increased carbon content $[\mathbf{1 0 , 2 1 ]}$. At higher carbon levels a transition to pearlite microstructure changes the pinning-domain structure interaction $[\mathbf{1 7 , 2 1}]$. Associated changes in microstructure complicate the interaction of domain structure with carbon content (pinning sites), followed by a plateau and a reduction of MBN at higher stresses [16,21]. Materials having a higher carbon density show a later turn over [6]. In contrast, higher strength steels such as HY-80, which are also characterized by a high pinning site density, show a continuing increase with stress compared with 1095, 1018 and interstitial free (IF) steels, which have the lowest pinning density [6]. These studies are complicated by differences in material microstructure and the pinning site density that can only be inferred from carbon content.

Loss of toughness in low alloy steels can occur due to two thermal treatments, 1) holding or slow cooling the alloy steels, previously held in the temperature range 350 to $550^{\circ} \mathrm{C}$, which is known as temper embrittlement, where loss of strength at grain boundaries occurs due to segregated impurities such as ( $\mathrm{S}, \mathrm{P}, \mathrm{Sb}, \mathrm{Sn}$ etc.), and 2) tempering as-quenched alloy steels in the range 250 to $450{ }^{\circ} \mathrm{C}$, producing tempered martensite embrittlement [25]. Work conducted here at $525^{\circ} \mathrm{C}$ was performed in the range of temperatures that produce temper embrittlement.

A number of studies have examined effects of temper embrittlement on MBN [26-28]. Moorthy et al. [27] observed that the presence of two or more different types of carbide in tempered Cr-Mo ferritic steels substantially reduced Barkhausen emissions compared with growth of single carbides in carbon steel. Final mechanical properties of supermartensitic stainless steels have been adjusted by quenching and tempering heat treatments with MBN detecting onset of temper embrittlement at $500{ }^{\circ} \mathrm{C}$ [28]. Allison et al. [29] have examined magnetic and magneto-acoustic properties of cast HY-80 steel, observing an increase in number of large magnetoacoustic emission events. Application of magneto-acoustic remanence to determine degree of embrittlement has been patented [30]. Isothermal temper-embrittlement studies on 5140 steel using impact tests, metallographic examination, microhardness, and lattice parameter measurements have also been conducted [31].

Recent studies have examined stress dependence of HY-80 steel, combined with initial stages of isothermal tempering treatment at $525{ }^{\circ} \mathrm{C}$ up to 48 hours [32]. Isothermal tempering treatment at $525{ }^{\circ} \mathrm{C}$ from 48 hours to 336 hours results in temper embrittlement $[\mathbf{1 4}, \mathbf{3 3}]$. Measurements on cast HY-80 steel has shown that MBN decreases exponentially with heat treatment, with decay constants in the range of 160-190 hours [33]. Decrease in MBN signal has been associated with migration of impurity elements to grain boundaries where they can no longer directly pin the domain structure $[\mathbf{1 4}, \mathbf{3 3}]$.

This paper further develops the physical basis for Barkhausen noise generation under conditions of applied stress and in the presence of pinning. The interaction of domain structure with pining sites and consequent change in domain structure with stress is considered to outline an experimental process. The process is evaluated against stress dependence of Q1N steel, which is similar to HY-80 steel [34]. Temper embrittlement of Q1N steel is performed in order to modify pinning density within grains, without substantially changing the microstructure. The measured stress dependence of MBN is explained in terms of changes in domain structure, and its interaction with changing pinning site density is used to explain the observed reduction in stress dependence of MBN energy with increasing embrittlement. The potential broad application of the process is explored for a number of areas where magnetic measurements are performed.

\section{Theory}

The generation of Barkhausen noise is dependent on the existing domain structure and how it interacts with 1) microstructure, 2) domain energy landscape, which includes the presence of pinning sites within grains [1], and 2) internal sample field produced by neighboring grains [35]. The Magnetic Object (MO) model, first described by Berttoti $[\mathbf{3 6}, 37,38]$, considers regions of relatively independent magnetic behaviour. In its further development, these regions have been considered under non-magnetized conditions as flux closed domain structures arising within single grains where grain boundaries form the periphery of the MOs and, therefore, define the region in which the domain wall motion that 


\section{Advanced Materials Letters www. vbripress.com/aml}

generates Barkhausen noise occurs, as represented in Fig. 1. Under existing or applied stress conditions modification of the orientation and number of $180^{\circ}$ and $90^{\circ}$ domains, which minimize the total energy of the domain configuration, arises $[\mathbf{1 , 1 3}]$, as shown from Fig. 1(c) to Fig. 1(d) for a progressively increasing stress condition. Given typical steel conditions the distribution and number of pinning sites within each grain could be considered a constant [13]. However, with heat treatment, spheroidizing [10] can decrease the density and number of pinning sites within grains. More recent work has indicated the effective removal of pinning sites from within HY-80 grains, after $48 \mathrm{hrs}$ of isothermal tempering treatment at $525^{\circ} \mathrm{C}$, due to embrittlement $[\mathbf{3 2}, 33]$. Temper embrittlement results in reduction of fracture toughness of alloy steels when heated or slowly cooled through the embrittling temperature range and arises due to migration of impurity elements to grain boundaries [25]. The movement of impurity elements, which act as pinning sites for domain structures, facilitates a study of the interaction of domain structures with changes in pinning site density. Domain structures may also be modified by application of tensile elastic stress, which increases the number of $180^{\circ}$ domain walls within grains [13]. The model developed here considers the interaction of domain structures, which are modified by tensile stress, with a decrease in number of pinning sites with heat treatment, associated with temper embrittlement, and resultant MBN that may be generated. (a)

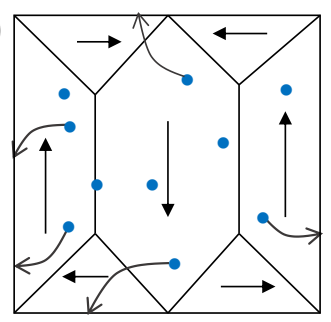

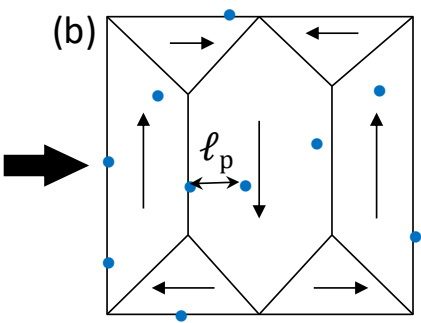
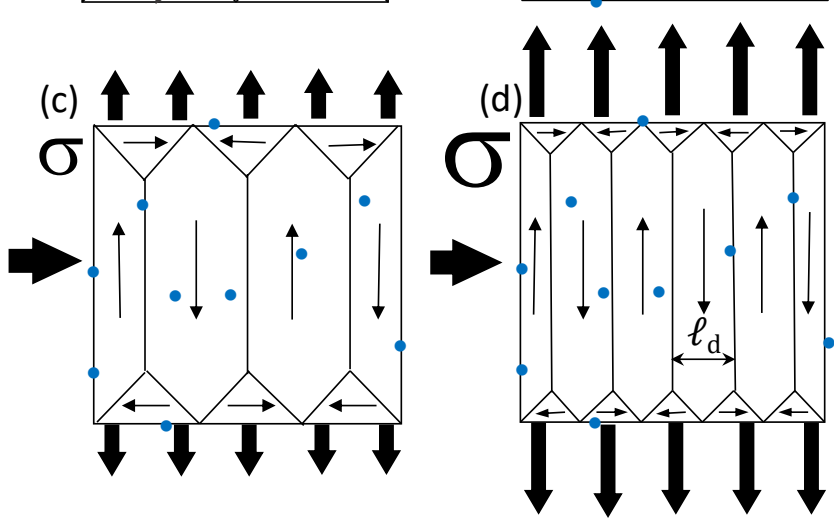

Fig. 1. Migration of pinning sites, indicated by solid circles (๑), from (a) within a magnetic object during isothermal heat treatment to grain boundaries in (b). Jump distance, $\ell_{\mathrm{p}}$, for $180^{\circ}$ domain wall for vertically applied field is shown in (b). Refinement of $180^{\circ}$ domain wall structure from (b) no stress condition to (c) with medium tensile uniaxial stress $(\sigma)$ and (d) high stress with pinning sites indicated by solid circles ( $\bullet$ ) or movement to nearest domain wall, $\ell_{\mathrm{d}}$, as indicated in (d).
Fig. 1 (a) to (b) shows a representation of migration of pinning sites due to impurity elements and/or carbides from within the MO towards the grain boundaries, due to heat treatment that results in temper embrittlement. The impurity element migration reduces the effective interaction of the domain structure with pinning sites and thereby, the magnetic Barkhausen noise signal $[\mathbf{3 2}, 33]$. The introduction of stress refines the domain structure, thereby increasing the number of potential Barkhausen events $[\mathbf{1 3 , 3 9 , 4 0}]$, as well as modifying the potential jump distances during magnetization.

As shown in Fig. 1 (c) and (d), the introduction of more $180^{\circ}$ domain walls by stress results in more opportunities for abrupt domain wall motion to pinning sites (compare Fig. 1 (b) and (c)), but at higher stresses for a finite number of pinning sites the domain wall refinement may exceed the pinning site density and domain walls, which are not pinned, will carry out the magnetization process, as shown in Fig. 1 (d) for a domain wall movement without a pinning site over a distance, $\ell_{\mathrm{d}}$, without an associated Barkhausen event. As a consequence, Barkhausen noise is expected to be reduced.

\section{Experimental}

The study was conducted with Q1N steel, which is similar to HY-80 steel, having a minimum yield strength of 550 $\mathrm{MPa}$. Samples were obtained from the same source as those in Ref. [41], where chemical specifications are also given. Cast plate Q1N samples had been ground smooth and were stress relieved. Samples that were $33 \mathrm{~mm}$ wide $3.1 \mathrm{~mm}$ thick and $177.5 \mathrm{~mm}$ long were heat treated at 96 hours, 168 hours and 336 hours to induce different degrees of temper embrittlement. Thereby, decreasing pinning site density within grains by inducing migration of pinning elements (impurities/carbides) towards grain boundaries $[32,33]$ as shown in Fig. 1(a). Samples were then stressed in tension using an Instron and MBN measurements were performed at successive loading steps using a dipole MBN probe details of which are provided in Refs. [14,21]. Microstructural characterization was done by scanning electron microscope.

The MBN dipole probe, used to perform Barkhausen noise measurements consisted of excitation coils mounted on the poles of a U-Core electromagnet, with a second set of coils at the pole ends to monitor the flux in the circuit. One pickup coil, with axis normal to the sample surface, was mounted between the pole ends. The dipole probe was placed at the centre of the sample in such a way that the 50 $\mathrm{Hz}$ excitation field was aligned with the applied tensile stress direction, which was also the rolling direction (RD). The flux was increased from $0.1 \mathrm{~T}$ to $1 \mathrm{~T}$ using flux control at each of the poles [42] and resulting MBN energy, which is given by the integral of the voltagesquared signal over time, summed over all of the Barkhausen events with in a cycle, given as $[\mathbf{1 , 3 5}]$

$$
\mathrm{MBN}_{\text {Energy }}=\sum_{\text {events }} \int \mathrm{V}^{2} \mathrm{dt},
$$




\section{Advanced Materials Letters www. vbripress.com/aml}

was evaluated. Stress dependent measurements were for stresses up to $450 \mathrm{MPa}$ for the four heat treated samples.

\section{Results and discussion}

Microstructure of Q1N steel, shown in Fig. 2, was examined using a scanning electron microscope (SEM). The sample was prepared by standard metallographic procedures for microstructural analysis [43]. Tempered martensitic microstructure with fine carbides distributed throughout the matrix was observed, which is characteristic microstructure of Q1N steel. Scanning electron microscope (SEM) examination revealed that tempered martensite structure (ferrite + cementite) was present. Thickness of ferrite plates was about $1.5 \mu \mathrm{m}$ and carbides were about $100-150 \mathrm{~nm}$ in diameter. Isothermal heat treatment lead to modification of many ferrite plates into equiaxed ferrite grains with $8 \mu \mathrm{m}$ size. Additionally, carbides were agglomerated and increased in size by up to $300 \mathrm{~nm}$. These carbides were observed to migrate towards the grain boundaries with increase in holding time from 0 and 96 hours, as shown in Fig. 2. This resulted in a decrease in carbide density within grains with time as has also been observed for HY-80 steel $[\mathbf{3 2}, \mathbf{3 3}]$.

(a)

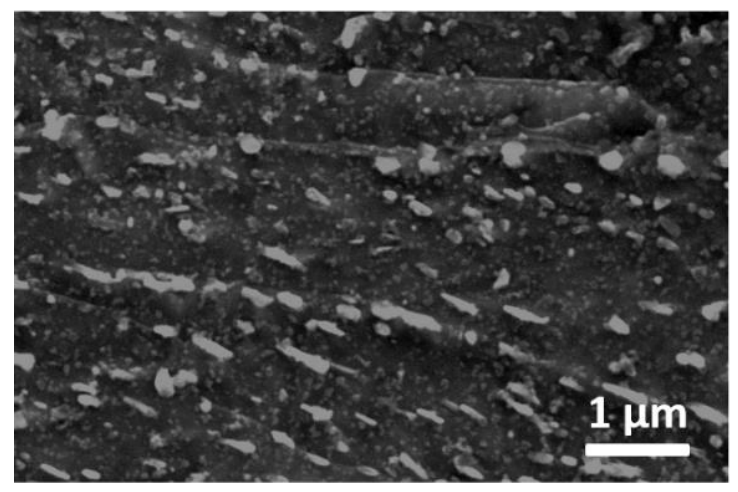

(b)

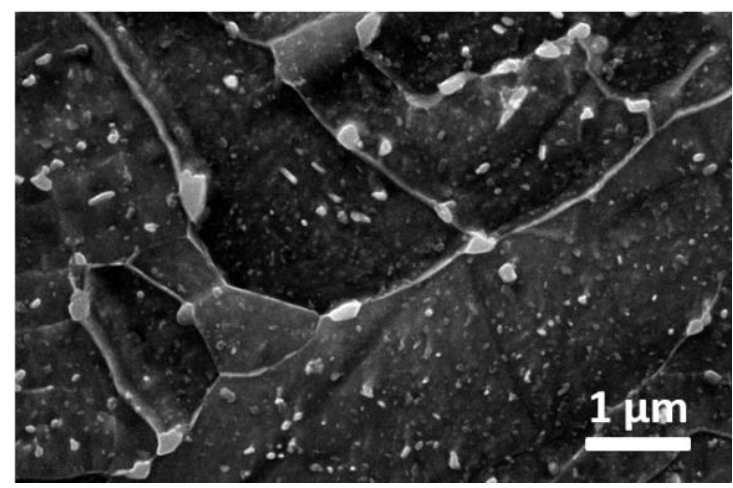

Fig. 2. SEM images of migration of carbides towards grain boundaries in Q1N steel, between (a) 0 hours and (b) 96 hours during isothermal temper embrittlement at $525{ }^{\circ} \mathrm{C}$, reduces potential interaction with ferromagnetic domain structures within grains during magnetization.

Draper and Ankem [44] observed that within the temperature embrittlement range of $\mathrm{HY}-80$ at $482{ }^{\circ} \mathrm{C}$, numerous carbides were observed to form at prior austenite grain boundaries, resulting in embrittlement carbide coarsening and/or nucleation, which was identified as $\mathrm{M}_{23} \mathrm{C}_{6}$. Little change in optical microstructure was observed. These results are consistent with observations and inference of change in pinning site density within grains made here.

MBN tests were performed on Q1N samples for different tempering times and therefore, varying density of pinning sites. Measurements were performed at various flux sweep rates as a function of tensile stress as shown in Fig. 3a to Fig. 3d. In general, MBN energy was observed to increase with stress along the rolling direction (RD), but amount and rate of increase changed with tempering time. For the 0 hour sample, MBN energy shows a monotonic increase, with increasing peak flux density (also equivalent to flux rate), but with rate of change slowing at higher peak fluxes. With increasing stress the MBN energy increases at all excitation flux rates, in agreement with models that associate increased MBN energy with increases in the $180^{\circ}$ domain wall population $[\mathbf{1 , 1 2 , 1 3}$. Note that for highest stresses MBN energy increases more rapidly between 0.2 and 0.4 Tesla, followed by a linear increase in Fig. 3a with flux density thereafter. Rate of increase with flux above 0.4 Tesla appears constant for the various stress levels, but with higher levels having been achieved for higher stresses. In terms of the model presented here, the pinning density within grains for the 0 hour sample is maximized and application of tensile stress increases the $180^{\circ}$ domain wall population, which increases the total number of potential interactions with pinning sites and therefore, the total MBN energy.
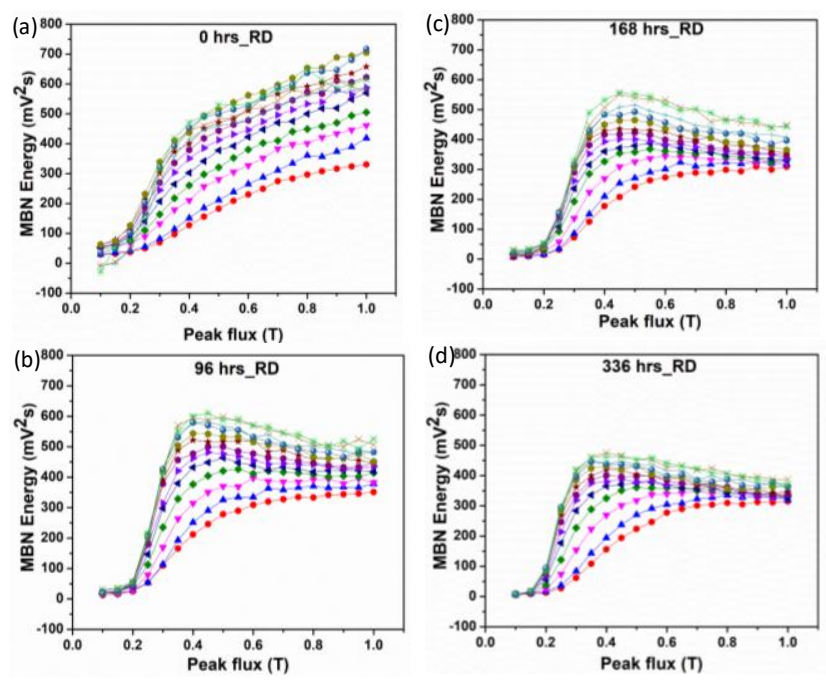

Fig. 3. MBN energy as a function of peak flux at various stress levels for (a) non-heat treated sample and samples heat treated at $525^{\circ} \mathrm{Cat}$ (b) 96 hours, (c) 168 hours and (d) 336 hours. Stress levels symbols are: $0 \mathrm{MPa}$ $(\bullet), 40 \mathrm{MPa}(\boldsymbol{\Delta}), 80 \mathrm{MPa}(\boldsymbol{\nabla}), 120 \mathrm{MPa}(\diamond), 160 \mathrm{MPa}(\varangle), 200$ $\mathrm{MPa}(\triangleright), 240 \mathrm{MPa}(\bullet), 280 \mathrm{MPa}(\star), 320 \mathrm{MPa}(\star)), 360 \mathrm{MPa}(\bullet)$, $400 \mathrm{MPa}(+), 440 \mathrm{MPa}(\times)$, and $450 \mathrm{MPa}(*)$.

After substantial temper embrittlement has been introduced, in this case for 96, 168 and 336 hour tempering times, obvious changes in the flux density dependent MBN energy behaviour are observed, shown in 


\section{Advanced Materials Letters www. vbripress.com/aml}

Fig. 3b, Fig. 3c and Fig. 3d, respectively. A similar increase of MBN energy with peak flux is observed up to 0.4 Tesla, but now with steadily decreasing levels of maximum MBN energy with increased holding time at constant stress as shown in Fig. 4 for $450 \mathrm{MPa}$. Here, the overall reduction in MBN energy with holding time is attributed to a reduction in domain wall pinning sites as previously reported in Refs. [32,33]. In contrast to the continuing increase of MBN energy with peak flux up to $1.0 \mathrm{~T}$, observed for the 0 hour sample, temper embrittled samples demonstrate a decrease from the peak value at $0.4 \mathrm{~T}$ and maximum tensile stress of $450 \mathrm{Mpa}$. In terms of effects considered here, this reflects magnetization processes at higher flux rates and stress levels, which produce a greater number of $180^{\circ}$ domain walls, but involve fewer abrupt domain wall motions due to reduced density of pinning sites and therefore, result in reduced MBN energy signal response.

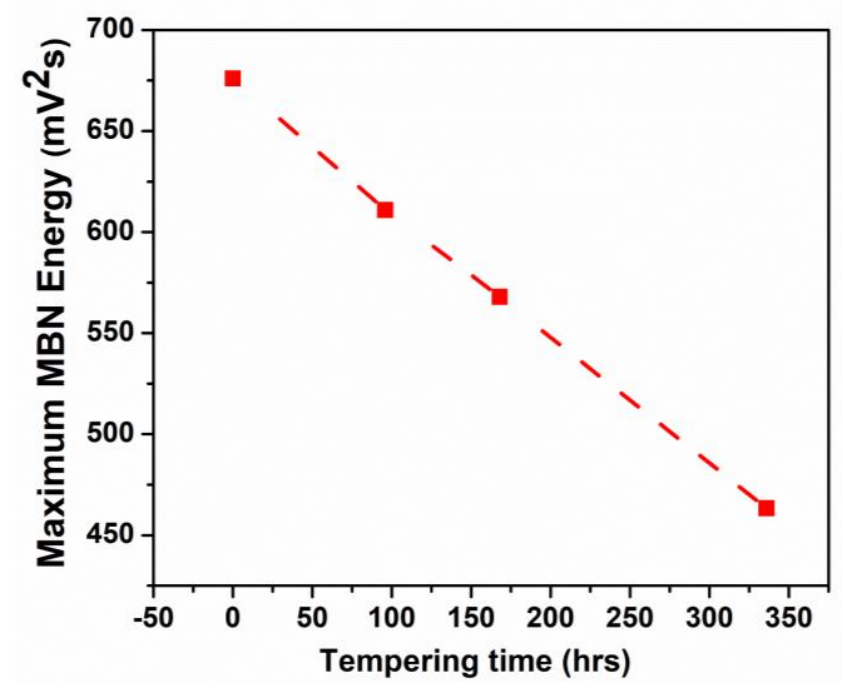

Fig. 4. Maximum MBN energy as a function of tempering time at 450 MPa.

The model of domain wall structure interactions with pinning sites presented here qualitatively describes the behaviour of MBN energy with increasing stress and removal of intragranular pinning sites by temper embrittlement. Detailed examination of specific mechanisms underlying domain magnetization processes, for example the increase in MBN energy up to 0.4 Tesla and its behaviour up to $1.0 \mathrm{~T}$ are left for future development of more advanced domain magnetization models that incorporate dynamic domain structure interactions with intragranular pinning sites.

The experimental process presented in this paper points towards development of an advanced understanding of changes in domain structure with applied stress and consequent interactions with pinning within grains as measured by magnetic Barkhausen noise. It is anticipated that the control of pinning sites for domain structures within ferromagnetic materials will have implications for optimization of magnetization processes, as required for electrical steels $[\mathbf{4 , 5}]$ and for non-destructive characterization of steel structures in oil and gas pipelines [2], naval structures [6] and aircraft landing gear [7]. The goal is to develop models that will provide information on MBN response for particular materials so that the state of stress and temper embritllement may be nondestructively assessed.

\section{Conclusion}

This paper outlined progress towards a physics based model that described Barkhausen noise response of steel materials undergoing magnetization, with particular consideration of domain structure in terms of number of $180^{\circ}$ domain walls as modified by the application of tensile stress and interaction with pinning sites within grains, as provided by impurity elements and carbides, whose density was varied by isothermal heat treatment. Implications for this work are the development of more complete magnetization models that incorporate domain structure interactions with pinning. Practical applications include non-destructive characterization of the state of temper embrittlement and stress in high strength steel materials using magnetic Barkhausen noise measurements and an improved understanding of magnetic processes in steels. The work will facilitate the potential of MBN sensing technology for advanced monitoring of steel structure condition.

\section{Acknowledgements}

The first author would like to thank S. Farrell of Defence Research Development Canada (DRDC) for providing the Q1N samples used in the study. This work was supported in part by DRDC and in part by Natural Sciences and Engineering Research Council of Canada (NSERC).

\section{Author's contributions}

TK developed the theory: AS and RU performed the experiments: AS and TK performed the data analysis: TK wrote the paper: (TK, AS, RU are the initials of authors). Authors have no competing financial interests.

\section{Keywords}

Magnetic Barkhausen Noise; Domain Wall Pinning; Domain Structure, Hardness.

\section{Received: 31 October 2019}

Revised: 23 December 2019

Accepted: 31 December 2019

\section{References}

1. Krause, T. W.; Samimi, A.; ASM Handbook Volume 17 Nondestructive evaluation of materials, Tenth Edition, ASM Int., Materials Park, Ohio, 2018. 515-530.

2. Krause, T. W.; Mandal, K. ; Hauge, C. ; Weyman, P.; Sijgers, B.; Atherton D. L.; Journal of Magnetism and Magnetic Materials, 1997, 169, 207.

3. Desjardins, D. P. R.; Krause, T. W.; Clapham L.; Nondestructive Testing and Evaluation Int., 2016, 80, 65.

4. Samimi, A. A.; Krause, T. W.; Clapham L.; Gallaugher; Ding, M. Y. ; Ghosh, P.; Chromik, R.; Knight, A. M.; J. Nondestr. Eval., 2014, 33, 663. 


\section{Advanced Materials Letters www. vbripress.com/aml}

5. Krause, T. W.; Szpunar, J. A.; Birsan, M,; Atherton D. L.; J. Appl. Phys., 1996, 79, 3156.

6. Samimi, A. A.; Krause, T. W.; Clapham L.; J. Nondestr. Eval., 2016, 35, 32 .

7. Morita, R.; Kobayashi, S.; Odeshi, A. G.; Szpunar, J. A.; Miura, K.; Kamada, Y.; AIP Advances, 2016, 6, 055903.

8. Cullity, B. D.; Introduction to Magnetic Materials, AddisonWesley, Reading, Massachusetts, USA, 1972.

9. Pérez-Benitez, J. A.; Capó-Sánchez, J.; J. Appl. Phys., 2008, 103, 043910.

10. Inaguma, T.; Sakamoto, H.; Hasegawa, M.; IEEE Trans. Magn, 2013, 49, 1310.

11. Karjalainen L.P.; Moilanen, M.; NDT Int., 1979, 12, 51.

12. Krause, T. W.; Pulfer, N.; Weyman, P.; Atherton D. L.; IEEE Trans. Magn., 1996, 32, 4764

13. T. W. Krause, Clapham L.; Pattantyus, A.; Atherton, D. L.; J. Appl. Phys., 1996, 79, 4242.

14. Saleem, A.; Underhill, P. R.; Herve, N. ; Farrell S. P.; Krause, T. W.; Quant. NDE, AIP Conf. Proc., 2102, 080009, 2019.

15. Pasley, R.L., Materials Evaluation, 1970, 28, 157.

16. Gauthier, J.; Krause, T. W.; Atherton D. L.; NDT \& $E$ International, 1998, 31, 23.

17. Alam-Samimi, A.; "Magnetic Barkhausen Noise Testing: Steel Grades and Stress Response," PhD Dissertation, Dept. of Physics, Engineering Physics and Astronomy, Queen's University, 2015.

18. Titto, S.; Otala, M.; Säynäjäkangas, S.; Non-Destructive Testing, 1976, 9, 117.

19. Clapham L.; Jagadish, C.; Atherton D. L.; Acta Metallurgica et Materialia, 1991, 39, 1555.

20. Gallaugher, M.; Samimi, A.; Krause, T. W.; Clapham L.; Chromik, R.R.; Metallurgical and Materials Transactions A, 2015, 46A, 1262.

21. Samimi, A. A.; Krause, T. W.; Clapham L.; J. Nondestr. Eval., 2016, 35,40 .

22. Hsu, A.; "Stress Effects on Magnetic Barkhausen Noise Measurements from Magnetized Pipeline Steel," Ph.D. Dissertation, Queen's University, 2000.

23. Dhar A.; Atherton D. L.; IEEE Trans. Magn., 1992, 28, 3363.

24. Mandache, C.; Krause, T. W.; Clapham L.; IEEE Trans. Magn., 2007, 43, 3976.

25. Horn, R.M.; Hutchie, R.O.; Metallurgical Transactions A, 1978, 9A, 1039.

26. Raj, B.; Moorthy, V.; Vaidyanathan, S.; Materials Evaluation, 1997, 55, 1, 81 .

27. Moorthy, V.; Vaidyanathan, S.; Jayakumar, T.; Raj, B.; Philosophical Magazine A, 1998, 77, 6, 1499,

28. Tavares, S. S. M.; Noris, L. F.; Pardal, J. M.; da Silva, M. R.; Engineering Failure Analysis, 2019, 100, 322.

29. Allison, S. G.; Yost, W. T.; Cantrell, J. H.; Hasson, J. H.; Nondestructive Evaluation of Temper Embrittlement in HY80 Steel,

https://lib.dr.iastate.edu/cgi/viewcontent.cgi?article=1681\&context =qnde

30. Allison, S. G.; Namkung, M.; Yost, W. T.; Cantrell, J. H.; U.S. Patent 5121058, 1992.

31. Bush, S. H.; Siebert, C. A.; Journal of Metals, 1954, 6, 11, 1269.

32. Saleem, A.; Swanson, D.; Underhill, P. R.; Farrell, S. P.; Krause, T. W.; "Affect of Temper Embrittlement and Stress on Magnetic Barkhausen Noise of Rolled HY-80 Steel", 13 ${ }^{\text {th }}$ International Conference on Barkhausen noise and micromagnetic testing, (ICBM12), 23-26 Sep., Prague, Czech Republic, 2019.

33. Saleem, A.; Underhill, P. R.; Farrell, S. P.; Krause, T. W.; IEEE Trans. Magn., 2019.

34. Farrell S. P.; MacGregor, L. W.; Porter, J. F.; Powder Diffraction 2010, 25, 119.

35. Krause, T. W.; Clapham L.; Atherton, D. L.; J. Appl. Phys., 1994, $75,7983$.

36. Bertotti, G.; J. Appl. Phys., 1985, 57, 2110.

37. Bertotti, G.; J. Appl. Phys. 1983, 54, 5293.

38. Bertotti, G.; J. Appl. Phys., 1984, 55, 4339.

39. Dijkstra L.J.; Martius, U.M.; Review of Modern Physics, 1953, 25, 146.
40. Perevertov O.; Schaefer, R.; J. Phys. D: Appl. Phys., 2014, 47, 185001

41. Farrell, S. P. MacGregor, L. W.; "Residual stress analysis of Q1N submarine pressure hull steel with the portable miniature X-ray diffractometer," Internal Report of Defence Research and Development Canada_Atlantic_DRDC Atlantic TM, Dartmouth, NS, 2008. Accessed Oct. 292019. http://cradpdf.drdc-rddc.gc.ca/PDFS/unc104/p530777_A1b.pdf

42. White, S.; Clapham L.; Krause, T. W.; IEEE Trans. Instr. and Meas., 2012, 61, 1896.

43. ASTM International. E3-11(2017) Standard Guide for Preparation of Metallographic Specimens. West Conshohocken, PA; ASTM Int., 2017.

44. Draper, M.; Ankem, S.; J. Mater Sci., 2019, 54, 2601. 